\title{
Analysis of Mitochondrial Fatty Acid Oxidation Intermediates by Tandem Mass Spectrometry from Intact Mitochondria Prepared from Homogenates of Cultured Fibroblasts, Skeletal Muscle Cells, and Fresh Muscle
}

\author{
TIINA TYNI, MORTEZA POURFARZAM, AND DOUGLASS M. TURNBULL \\ Department of Neurology, University of Newcastle upon Tyne, Newcastle upon Tyne, NE2 4HH, U.K. \\ [T.T., D.M.T.]; Sir James Spence Institute of Child Health, Royal Victoria Infirmary, University of \\ Newcastle upon Tyne, Newcastle upon Tyne, NE1 4LP, U.K. [M.P.]; Hospital for Children and
}

Adolescents, 00029 HUS, Helsinki, Finland [T.T.] \begin{abstract}
ABST
Defects of mitochondrial fatty acid $\beta$-oxidation are an impor-
tant group of inherited metabolic disorders in children. Despite improved screening opportunities, diagnosis of these disorders is not often straightforward and requires enzyme analyses. Because therapy is effective in many of these disorders, rapid diagnosis is essential. We report a technique that allows analysis of fatty acid oxidation not only in cultured cells (fibroblasts, myoblasts, and myotubes) but also in fresh muscle homogenate. Fatty acid oxidation analysis was performed by incubating fresh muscle homogenate or harvested cultured cells with stable isotopically labeled palmitate. The intermediates generated were analyzed by tandem mass spectrometry. Results of patients with seven different $\beta$-oxidation disorders were compared with controls. Acylcarnitine intermediates in patient samples could be easily differentiated from the control samples. The acylcarnitine profile of each $\beta$-oxidation defect was compatible with localization of the enzyme defect. Both in patient and control samples, the same pattern of intermediates could be detected in fibroblasts, muscle
\end{abstract}

cells, and fresh muscle homogenate. The procedure described allowed correct diagnosis of all the $\beta$-oxidation defects studied. Utilization of fresh muscle samples reduces the delay in diagnosis related to tissue culture and is useful in diagnostic of patients with neuromuscular phenotype. Measurement of fatty acid oxidation intermediates from myoblasts or myotubes is an additional tool in investigating pathogenetic mechanisms of myopathy in $\beta$-oxidation defects. (Pediatr Res 52: 64-70, 2002)

CPT, carnitine palmitoyltransferase

LCHAD, long-chain 3-hydroxyacyl- CoA dehydrogenase

MAD, multiple acyl-CoA dehydrogenase

MCAD, medium-chain acyl-CoA dehydrogenase

MEM, minimum essential medium

MS/MS, tandem mass spectrometry

TFP, mitochondrial trifunctional protein

VLCAD, very long-chain acyl-CoA dehydrogenase
Defects of mitochondrial fatty acid $\beta$-oxidation are an important, and often treatable, group of inherited metabolic defects. The $\beta$-oxidation pathway involves several enzymes, and defects of most of these enzymes have been identified. Clinical manifestations of $\beta$-oxidation defects are relatively uniform, and thus the clinical features of a patient with a $\beta$-oxidation disorder seldom suggest a particular enzyme defect. Only three of up to 20 of these disorders, namely CPT II, LCHAD, and MCAD deficiencies, are caused by a single prevalent mutation.

Received March 19, 2001; accepted December 31, 2001.

Correspondence and reprint requests: Tiina Tyni, Hospital for Children and Adolescents, University of Helsinki, P.B.O 280, FIN-00029 HUS, Helsinki, Finland; e-mail: Tiina.Tyni@hus.fi

Supported by the Juselius Foundation, the Arvo and Lea Ylppö Foundation, the Ulla Hjelt Trust, The Pediatric Research Foundation, Finland, and the Wellcome Trust, U.K.
To reduce the need for enzyme assays in diagnosis of $\beta$-oxidation defects, there has been interest in developing a method capable of localizing the site of the enzyme defect in the $\beta$-oxidation pathway. Analysis of acylcarnitine intermediates from blood samples is the most widely used screening test for $\beta$-oxidation defects. However, it is not always diagnostic during a stable phase of the disease (1). Incubation of cultured cells with palmitate and carnitine and analysis of acylcarnitine intermediates is another possible technique for localizing the site of the enzyme defect. In the first published method, cultured skin fibroblasts were incubated with radiolabeled palmitate and intermediates were analyzed by HPLC $(2,3)$. Introduction of MS/MS provided the opportunity to use nonlabeled or stable isotope-labeled palmitate in these analyses. So far, methods using stable 
isotopes have been used only when incubating cultured cells on monolayers (4-7).

Although $\beta$-oxidation defects usually present in infancy, neuromuscular presentation is often seen later in life. Adolescents and adults with muscle weakness, muscle pain, or myoglobinuria are an important group of patients, in whom a $\beta$-oxidation defect is often a possibility, but only one among several other diagnoses. Initial screening tests in patients suspected to have a $\beta$-oxidation defect include analysis of blood acylcarnitines and the common mutation of CPT II deficiency. If these tests are negative, the next logical step in the diagnosis is muscle biopsy. Incubation of fresh muscle sample with palmitate would reduce the diagnostic delay caused by the time required to establish the culture of cells. In addition to using fibroblasts and fresh muscle for diagnostic studies, we also wished to study fatty acid oxidation in cultured muscle cells, which could potentially be an ideal cell culture system to study mechanism of muscle changes in defects of fatty acid $\beta$-oxidation. We report a technique that would allow diagnosis of $\beta$-oxidation defects not only in cultured cells but also in fresh muscle homogenate.

\section{MATERIALS AND METHODS}

Subjects. Skin fibroblasts were obtained from seven subjects with no evidence of an inborn error of mitochondrial fatty acid oxidation and 15 patients with a proven disorder of mitochondrial fatty acid oxidation (one patient with CPT I deficiency, three with CPT II deficiency, two with VLCAD deficiency, three with LCHAD deficiency, three with mitochondrial TFP deficiency, two with MCAD deficiency, and one with MAD deficiency). Patients with LCHAD deficiency were children with the prevalent homozygous mutation, G1528C, of LCHAD deficiency. Out of three adult patients with TFP deficiency, one patient had a severely reduced amount of immunoreactive protein and two were heterozygous for the G1528C mutation and had reduced activities of all TFP enzymes. In patients with CPT I and II deficiencies, the diagnosis was made by measurement of enzyme activities (8). Two of the patients with CPT II deficiency were heterozygous for the prevalent mutation, C439T, of CPT II deficiency. Diagnosis of the patients with MCAD deficiency was confirmed by measuring enzyme activity and detection of G985A mutation. In both adult patients with VLCAD deficiency, the diagnosis was confirmed either by mutation analysis or enzyme assay. The diagnosis of the patient with MAD deficiency was based on glutaric aciduria and characteristic pattern of intermediates in blood acylcarnitine analysis. The disease was not riboflavin responsive. Altogether, one patient with CPT I deficiency, three patients with LCHAD deficiency, two patients with MCAD deficiency, and one patient with MAD deficiency had an early-onset disease.

Muscle biopsy was obtained from seven control subjects with no evidence of an inborn error of mitochondrial fatty acid oxidation, and four adult patients with a $\beta$-oxidation disorder (one with TFP deficiency, one with CPT II deficiency, one with VLCAD deficiency, and another with MAD deficiency). The muscle biopsy was obtained from the vastus lateralis muscle. Fatty acid oxidation analysis was performed from the fresh biopsy specimen of four controls studied, and primary myoblast culture was established from the biopsy specimen of the remaining three subjects. Both myoblast culture and fatty acid incubation from fresh muscle could be performed from the muscle sample of the patient with TFP deficiency. Myoblast cultures were obtained from the samples of patients with VLCAD and CPT II deficiencies. From the sample of the patient with MAD deficiency, only fresh muscle incubation was performed. Fatty acid oxidation was also analyzed from cultured fibroblasts from all of these patients.

All studies were approved by the institutional review board and all human studies were conducted with informed consent.

Culture of fibroblasts and myoblasts. Cell culture reagents were purchased from Invitrogen (Paisley, Scotland, U.K.) except for MEM- $\alpha$ and chick embryo extract, which were supplied by Sigma Chemical (Poole, U.K.) and Imperial (Andover, U.K.), respectively. The plasticware was purchased from Greiner Labortechnik (Gloucestershire, U.K.).

Fibroblast cultures were established from a skin biopsy with conventional methods. Fibroblasts were cultured in MEM supplemented with $10 \%$ (vol $/ \mathrm{vol})$ fetal bovine serum, $1 \%$ (vol/vol) MEM essential amino acids, $1 \%$ (vol/vol) MEM vitamins, and $0.1 \mathrm{mg} / \mathrm{mL}$ streptomycin and $100 \mathrm{U} / \mathrm{mL}$ of benzylpenicillin. The fibroblasts were grown to confluence in two $75-\mathrm{cm}^{2}$ tissue culture flasks and harvested by trypsinization. No culture medium was added after trypsin treatment; instead, the trypsin was inactivated by diluting the solution with PBS.

Aneural muscle cultures were established by isolating satellite cells from human muscle biopsy specimens essentially as described earlier (9). Myoblasts were grown in F10 culture medium supplemented with $20 \%$ (vol/vol) fetal bovine serum, $1 \%$ (vol/vol) chick embryo extract, and antibiotics as above (10). Myoblasts were differentiated to myotubes using fusion medium: MEM- $\alpha$ supplemented with $2 \%$ (vol/vol) fetal bovine serum, $1 \%$ (vol/vol) chick embryo extract, $4 \mathrm{mM}$ L-glutamate, and antibiotics. F10 was replaced by the fusion medium when cells had reached $80 \%$ confluence. Fatty acid oxidation analysis of myotubes was performed when $>50 \%$ of nuclei were found within multinucleated cells (myotubes). Myoblasts and myotubes were harvested the same way as fibroblasts.

Preparation of cultured cells and skeletal muscle for incubation. The pellet of harvested cells was resuspended in 500 $\mu \mathrm{L}$ fatty acid incubation medium containing $110 \mathrm{mM} \mathrm{KCl}, 10$ $\mathrm{mM}$ N-2-hydroxyethylpiperazine-N'-2-ethanesulfonic acid buffer, $5 \mathrm{mM} \mathrm{MgCl} 2,10 \mathrm{mM}$ potassium phosphate, $1 \mathrm{mM}$ EGTA, $1 \mathrm{mM}$ ADP, $5 \mathrm{mM}$ ATP, $0.1 \mathrm{mM}$ CoA, $0.2 \mathrm{mg} / \mathrm{mL}$ cytomchrome $c$, and $2 \mathrm{mM}$ L-carnitine, $\mathrm{pH} 7.2$ (3). The resuspended pellet was then homogenized with a pellet pestle (Sigma Chemical) for $3 \times 30 \mathrm{~s}$ on ice. The cells were centrifuged at $17,000 \mathrm{~g}$ for $1 \mathrm{~min}$ and resuspended in $105 \mu \mathrm{L}$ of the incubation medium. A $20-\mu \mathrm{L}$ sample was taken for citrate synthase analysis.

The fresh muscle $(25-50 \mathrm{mg}$ ) was chopped in $1-1.5 \mathrm{~mL}$ (depending on the size of the biopsy) of incubation medium and homogenized with Ultra-Turrax T25 (IKA-Labortechnik, Jencons-PLS, Leighton, U.K.) at $8500 \mathrm{rpm}$ for $6 \mathrm{~s}$. The sample was centrifuged ( $600 \mathrm{~g}$ for $6 \mathrm{~min}$ ). The supernatant was taken 
and centrifuged at 17,000 $g$ for $6 \mathrm{~min}$. The pellet was resuspended with Socorex micropipette (CAMLAB, Cambridge, U.K.) in $220 \mu \mathrm{L}$ incubation medium. A $80 \mu \mathrm{L}$-sample was taken for citrate synthase measurement.

Incubation of muscle and fibroblasts. After a 5-min preincubation at $30^{\circ} \mathrm{C}$ in a shaking water bath, the reaction was started by adding $15 \mu \mathrm{L}$ (cell homogenate) and $40 \mu \mathrm{L}$ (muscle homogenate) of $2 \mathrm{mM}\left[\mathrm{U}-{ }^{13} \mathrm{C}\right]$-labeled palmitate (Cambridge Isotope Laboratories, Andover, MA, U.S.A.) bound to BSA in a 5:1 molar ratio, which resulted in the final palmitate concentrations of $0.3 \mathrm{mM}$ and $0.44 \mathrm{mM}$, respectively. The final concentrations of $\mathrm{L}$-carnitine in the cell and muscle incubations were $1.8 \mathrm{mM}$ and $1.72 \mathrm{mM}$, respectively. Samples $(20 \mu \mathrm{L}$ from the cell and $40 \mu \mathrm{L}$ from the muscle incubation, respectively) were taken after 30,60, and 90 min incubation. An additional sample was taken after 120 min incubation of muscle homogenate. The reaction was quenched using $5 \mu \mathrm{L}$ (per 20/40 $\mu \mathrm{L}$ sample) $1 \mathrm{M} \mathrm{H}_{2} \mathrm{SO}_{4}$. The samples were flash frozen in liquid nitrogen and stored short term at $-20^{\circ} \mathrm{C}$.

To optimize the homogenization procedure at the preliminary phase of this study, intactness of mitochondria was determined by measuring lactate dehydrogenase (cytosolic enzyme) and citrate synthase (enzyme of mitochondrial matrix) with standard methods (11). Throughout the study, intensities of acylcarnitine intermediates were referenced to intensities of internal standards and citrate synthase activity.

Extraction of acylcarnitine intermediates. Equal volumes of incubation sample ( $10 \mu \mathrm{L}$ for cells and $20 \mu \mathrm{L}$ for muscle) and internal standard mixture containing $50 \mu \mathrm{M}\left[{ }^{2} \mathrm{H}_{3}\right]$ acetylcarnitine, $5 \mu \mathrm{M}\left[{ }^{2} \mathrm{H}_{9}\right]$ octanoylcarnitine, and $5 \mu \mathrm{M}\left[{ }^{2} \mathrm{H}_{9}\right]$ palmitoylcarnitine were combined, and $200 \mu \mathrm{L}$ methanol was added. The sample was shaken vigorously for $30 \mathrm{~min}$, sonicated for $30 \mathrm{~min}$, and frozen at $-80^{\circ} \mathrm{C}$ for $30 \mathrm{~min}$. The sample was centrifuged $(17,000 \mathrm{~g}$ for $1 \mathrm{~min})$ and the supernatant was taken. The remaining pellet was re-extracted with $200 \mu \mathrm{L}$ methanol, sonicated, frozen down, and centrifuged as described above. To the combined supernatant, water was added to bring the methanol concentration to $80 \%$. The sample was then loaded to 2-cm DEAE Sephacel column (Amersham, Uppsala, Sweden) and washed through with $1.6 \mathrm{~mL} 80 \%$ methanol. Methanol was evaporated under the stream of nitrogen at $60^{\circ} \mathrm{C}$. After drying, the sample was derivatized with 75 $\mu \mathrm{L} 2 \mathrm{M} \mathrm{HCl}$-butanol for $15 \mathrm{~min}$ at $65^{\circ} \mathrm{C}$. Before the analysis by electrospray MS/MS, the derivatizing agent was removed with nitrogen and the butylated extracts were dissolved in 100 $\mu \mathrm{L} 70 \%$ acetonitrile in water.

Analysis of the intermediates. A Quattro II quadrupole MS/MS (Micromass, Altrincham, U.K.) equipped with an electrospray ion source and a Micromass Masslynx data system was used for the analysis of butylated acylcarnitines. The flow rate of the mobile phase ( $60 \%$ acetonitrile in water) was 30 $\mu \mathrm{L} / \mathrm{min}$. For all MS/MS experiments, argon was used as the collision gas at a pressure of $2 \times 10^{-3} \mathrm{mbar}$. A precursor ion scan of $\mathrm{m} / \mathrm{z} 85$ (10 scans of $4 \mathrm{~s}$ duration and $0.15 \mathrm{~s}$ interscan delay, collision energy $25 \mathrm{eV}$, cone voltage $30 \mathrm{~V}$ ) was used in the range of $\mathrm{m} / \mathrm{z} 250-550$ for the analysis of acylcarnitines.

\section{RESULTS}

Fibroblast incubations. Mean production of acetylcarnitine in control fibroblasts was $178.6,355.0$, and $484.7 \mu \mathrm{mol} / \mathrm{U}$ of citrate synthase activity after 30,60 , and $90 \mathrm{~min}$ incubation (SD 57.0, 107.3, and $166.6 \mu \mathrm{mol} / \mathrm{U}$ of citrate synthase activity, respectively) (Table 1). Acetylcarnitine production was lower in the patient group, with the mean of 55.6, 91.0, and 133.9 $\mu \mathrm{mol} / \mathrm{U}$ of citrate synthase activity (SD 39.7, 51.9, and 82.1 $\mu \mathrm{mol} / \mathrm{U}$ of citrate synthase activity), after 30,60 , and $90 \mathrm{~min}$ incubation, respectively. In samples taken after $60 \mathrm{~min}$ of incubation, there was no overlap between control and patient groups (range in controls 223.9-511.3 $\mu \mathrm{mol} / \mathrm{U}$ of citrate synthase activity and in patients $2.3-176.7 \mu \mathrm{mol} / \mathrm{U}$ of citrate synthase activity). In both patients with MCAD deficiency, acetylcarnitine production overlapped with that in controls after 30 min incubation but was lower than normal values after 60 and $90 \mathrm{~min}$ incubation (104.9 and $134.7 \mu \mathrm{mol} / \mathrm{U}$ of citrate synthase activity at $60 \mathrm{~min}$ and 218.5 and $172.6 \mu \mathrm{mol} / \mathrm{U}$ of citrate synthase activity at $90 \mathrm{~min}$ in patients versus $223.9-$ $511.3 \mu \mathrm{mol} / \mathrm{U}$ of citrate synthase activity at $60 \mathrm{~min}$ and $291.9-561.5 \mu \mathrm{mol} / \mathrm{U}$ of citrate synthase activity at $90 \mathrm{~min}$ in controls). Among all patients, reduction of acetylcarnitine production was less prominent in the patients with MCAD, CPT II, and LCHAD deficiencies.

Typical findings in patients with MCAD deficiency were very low levels of butyryl- and hexanoylcarnitines (undetectable at all three time points versus mean butyrylcarnitine 2.4 $\mu \mathrm{mol} / \mathrm{U}$ of citrate synthase activity and hexanoylcarnitine 9.6 $\mu \mathrm{mol} / \mathrm{U}$ of citrate synthase activity at $60 \mathrm{~min}$ in controls) (Fig. 1). Intermediates of the patient with CPT I deficiency showed uniformly low acylcarnitines, including hexadecanoylcarnitine, but hexadecanoyl/acetylcarnitine ratio was within normal range. In contrast, in CPT II deficient cell lines, acetylcarnitine production was low as well as most other short and mediumchain acylcarnitines, but a great quantity of hexadecanoylcarnitine remained. VLCAD-deficient cell lines showed low production of medium-chain intermediates and high hexadecanoyl/acetylcarnitine and long-chain/medium-chain acylcarnitine ratio $(\mathrm{C} 16 / \mathrm{C} 12$ range $44-255$ versus $3.76-9.72$ in controls). The most remarkable difference between patients with VLCAD and CPT II deficiencies was higher accumulation of tetradecanoylcarnitine and smaller $\mathrm{C} 16 / \mathrm{C} 14$ ratio in the former. Contrary to the monolayer incubations, C14:1 intermediate levels were not higher than in controls. The patient with MAD deficiency had low levels of all intermediates with carbon chain-lengths from $\mathrm{C} 2$ to $\mathrm{C} 14$, only $\mathrm{C} 14: 1$ and $\mathrm{C} 16: 1$ were within control values. Patients with LCHAD or TFP deficiency had relative accumulation of long-chain acylcarnitines and high 3-hydroxyhexadecanoylcarnitines (range 5.7$25.6 \mu \mathrm{mol} / \mathrm{U}$ of citrate synthase activity, control range $0-0.7$ $\mu \mathrm{mol} / \mathrm{U}$ of citrate synthase activity, and the range in cell lines with other $\beta$-oxidation defects $0-1.5 \mu \mathrm{mol} / \mathrm{U}$ of citrate synthase activity). Excluding controls and patients with LCHAD or TFP deficiency, 3-hydroxyhexadecanoylcarnitines were seen only in the patient with CPT I deficiency.

Muscle cell and fresh muscle incubations. The pattern of acylcarnitine intermediates was the same in fresh muscle and 
cultured muscle cell incubations and similar to that in fibroblast incubations both in control and patient samples (Table 2). Incubations of all the patient samples (from patients with CPT II, VLCAD, and TFP deficiencies) showed accumulation of long-chain acylcarnitines and other findings characteristic of each disorder described above.

In muscle incubations, acetylcarnitine production was higher in control samples compared with patient samples (Fig. 3) at all time points. After 60 min incubation, it ranged from 240.9 to $1058.2 \mu \mathrm{mol} / \mathrm{U}$ of citrate synthase activity in controls and 1.25 to $103.0 \mu \mathrm{mol} / \mathrm{U}$ of citrate synthase activity in the patients. The main accumulating intermediates in controls were hexanoyl-, dodecanoyl-, and tetradecanoylcarnitines (Fig. 2). In the patient with TFP deficiency, the major intermediates were tetradecanoyl-, hexadecanoyl, and 3-hydroxyhexadecanoylcarnitines. The ratio of 3-hydroxyhexadecanoyl-/ tetradecanoylcarnitine was 0.66 and 0.90 after $60 \mathrm{~min}$ and 90 min of incubation, respectively, in this patient, whereas in control samples the ratio was $<0.23$ up to 90 min incubation. In the patient with MAD deficiency, 3-hydroxyhexadecanoylcarnitine was undetectable. In control samples, the ratio of tetradecanoyl/decanoylcarnitine was $<20$ at all time points, whereas in the patients, the ratio was $>30$ at all time points. MAD and TFP deficiencies could be easily distinguished from each other by different overall profile of acylcarnitine accumulation and 3-hydroxyhexadecanoylcarnitine accumulation in TFP deficiency. In the muscle of the patient with MAD deficiency, acetylcarnitine production was severely reduced and accumulation of long-chain acylcarnitines was more pronounced than in the patient with TFP deficiency.

There was no clear difference in acylcarnitine profile between fibroblast incubations and myoblast and myotube incubations either in controls or patients. It was more difficult to optimize myotube incubations, because, in the cell lines with very large myotubes, the $\beta$-oxidation reaction could rapidly run out of the substrate.

\section{DISCUSSION}

The study showed that fatty acid oxidation intermediates generated by incubation of fresh muscle or cultured cells with stable isotope labeled palmitate and analyzed by MS/MS can be used for the diagnosis of defects in $\beta$-oxidation pathway. In this study, both cultured cells and muscle homogenates produced similar results. The acylcarnitine profiles produced were also characteristic for the disorder and could be used to localize the site of the enzyme defect in $\beta$-oxidation pathway. This suggests that these methods can be used as a screening test for a $\beta$-oxidation defect, especially when there is no blood sample available for acylcarnitine analysis.

Although several cell lines used in this study were from patients with a late-onset disorder and a relatively mild underlying $\beta$-oxidation defect, it was possible to distinguish patient cells lines from controls. The acylcarnitine profiles detected in our patient cell lines were compatible with the site of the enzyme defect causing accumulation of acylcarnitines proximal to the defect. The profiles of acylcarnitine intermediates detected in the patients with $\beta$-oxidation defects were very 

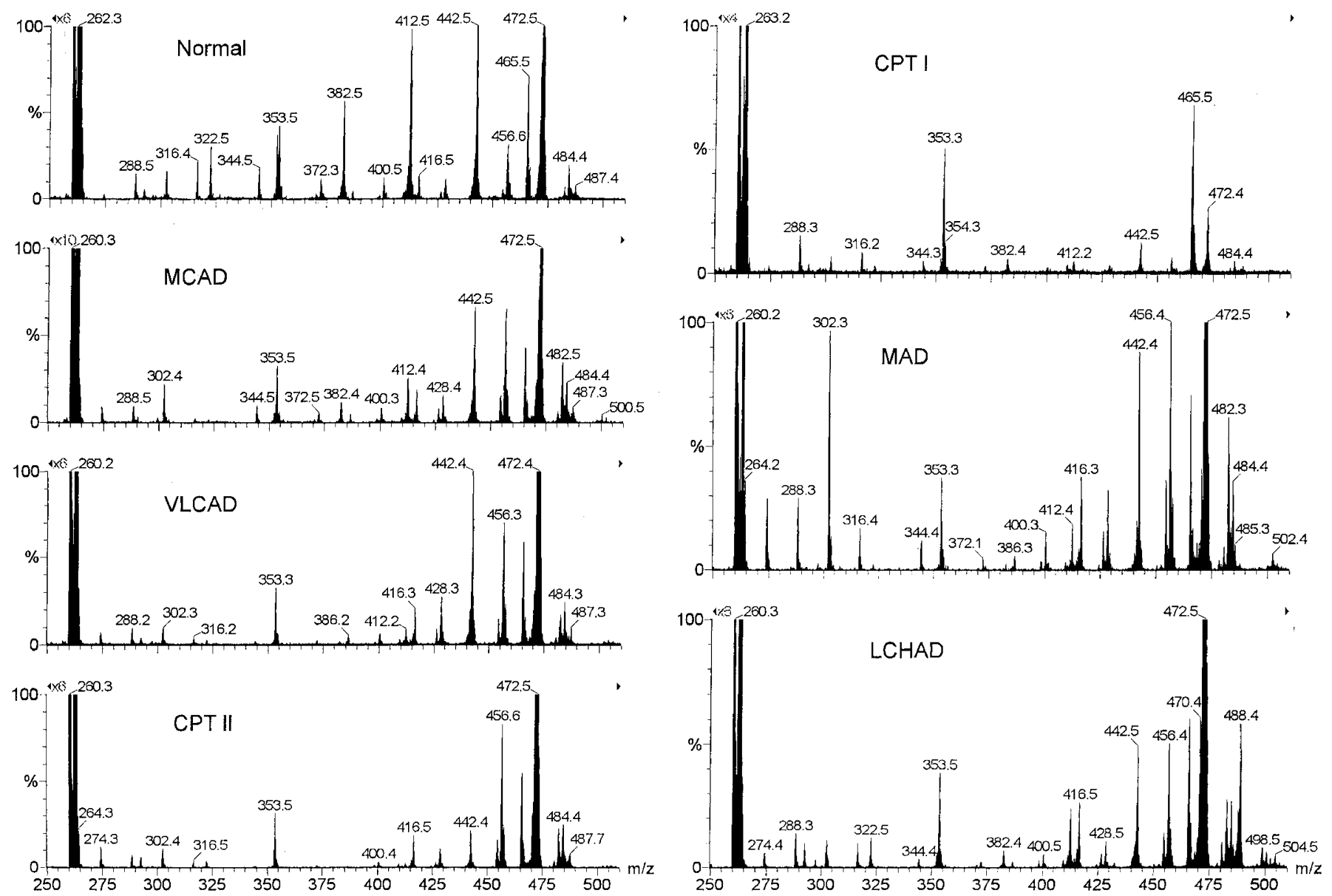

Figure 1. Acylcarnitine intermediate profiles generated by incubation of cultured fibroblasts with stable isotope-labeled palmitate and medium containing L-carnitine. The signals represent molecular ions of acylcarnitine butyl esters detected by MS/MS. $(A)$ Control; $(B)$ MCAD deficiency; $(C)$ VLCAD deficiency; $(D)$ CPT II deficiency; $(E)$ CPT I deficiency; $(F)$ MAD deficiency; and $(G)$ LCHAD deficiency. Internal standards: $\left[{ }^{2} \mathrm{H}_{3}\right]$-acetylcarnitine (m/z 263), $\left[{ }^{2} \mathrm{H}_{9}\right]$-octanoylcarnitine (m/z 353), $\left[{ }^{2} \mathrm{H}_{9}\right]$-palmitoylcarnitine (m/z 465). Stable isotope-labeled acylcarnitines: [U- $\left.{ }^{13} \mathrm{C}\right]$-acetylcarnitine $(\mathrm{C} 2)(\mathrm{m} / \mathrm{z} 262)$, [U- $\left.{ }^{13} \mathrm{C}\right]-$ butyrylcarnitine (C4) (m/z 292), [U- $\left.{ }^{13} \mathrm{C}\right]$-hexanoylcarnitine (C6) (m/z 322), [U- $\left.{ }^{13} \mathrm{C}\right]$-octanoylcarnitine $(\mathrm{C} 8)(\mathrm{m} / \mathrm{z} 352)$, [U- $\left.{ }^{13} \mathrm{C}\right]$-decanoylcarnitine $(\mathrm{C} 10)(\mathrm{m} / \mathrm{z}$ 382), $\left[\mathrm{U}_{-}{ }^{13} \mathrm{C}\right]$-dodecanoylcarnitine $(\mathrm{C} 12)(\mathrm{m} / \mathrm{z} 412)$, $\left[\mathrm{U}_{-}{ }^{13} \mathrm{C}\right]$-hydroxydodecanoylcarnitine $(\mathrm{C} 12-\mathrm{OH})(\mathrm{m} / \mathrm{z} 428)$, $\left[\mathrm{U}_{-}{ }^{13} \mathrm{C}\right]$-tetradecanoylcarnitine $(\mathrm{C}-14-\mathrm{OH})(\mathrm{m} / \mathrm{z}$ 442), [U- $\left.{ }^{13} \mathrm{C}\right]$-hexadecanoylcarnitine (C16) (m/z 472), [U- $\left.{ }^{13} \mathrm{C}\right]$-hydroxyhexadecanoylcarnitine (C16-OH) $(\mathrm{m} / \mathrm{z}$ 488). m/z, mass/charge. For incubation and analysis conditions see the text.

similar to those found in methods using radiolabeled palmitate and radio HPLC analysis $(2,3)$. It appears that although both monolayer incubations and analyses of harvested cells allow detection of most $\beta$-oxidation defects, the acylcarnitine profiles obtained from these assays are different. This is presumably due to different incubation times; the short incubation shows primary consequences of the block in the chain shortening of the substrate whereas accumulation of intermediates in longterm incubations also reflects the secondary effects of the enzyme defect.

The use of stable, isotopically labeled substrate and MS/MS analysis in fatty acid oxidation studies instead of radiolabeled palmitate or carnitine and HPLC analysis is naturally an advantage. Analysis of intermediates by the method of Ventura et al. (7) required two different analysis systems. In monolayer incubations, the volume of incubation medium is usually much larger than in our method, which increases the cost of the assay. Cold substrate, used mostly in monolayer incubation methods, reduces the cost but makes it more difficult to assess whether the incubation has been optimal because, according to our experience, long incubation times (72-96 h) may result in running out of the substrate in control cell lines. Medium containing palmitate used in monolayer incubations is not an optimal culture medium and may cause cell degeneration, especially when incubating cells with a $\beta$-oxidation defect. The use of stable isotope and relatively larger volume of the incubation medium in the method published by Ventura et al. (7) may resolve these problems. In that method, the number of incubated cells was small $\left(3.8 \mathrm{~cm}^{2}\right)$ compared with $2 \times 75 \mathrm{~cm}^{2}$ in our method. A small number of incubated cells is advantageous when performing analysis for prenatal diagnosis, but may make the method less reliable. Presumably, it is also necessary to analyze larger number of cells when comparing fatty acid oxidation in cell lines derived from different tissues. The ease of taking samples at different time points in our method improves the timing of the analysis. Homogenization 


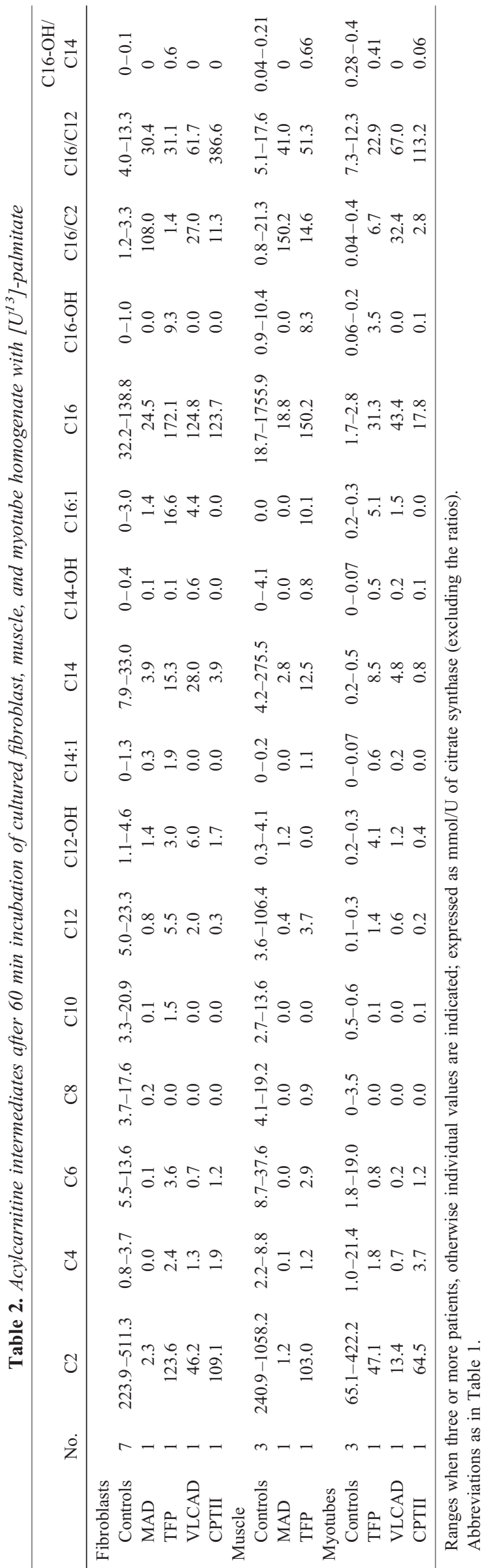

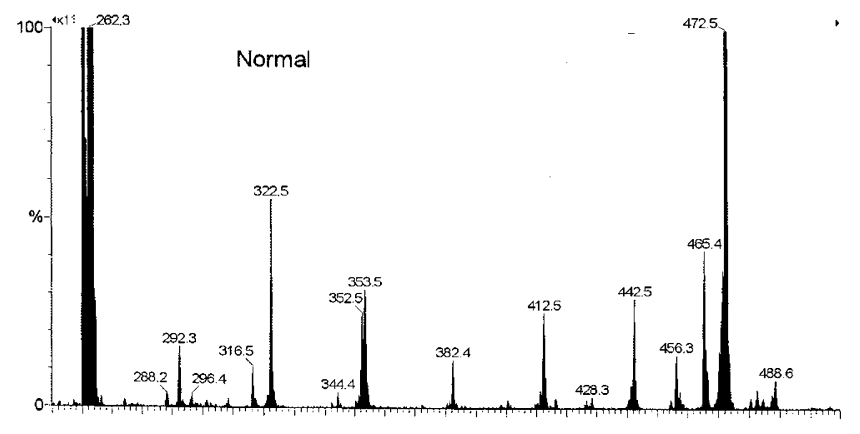
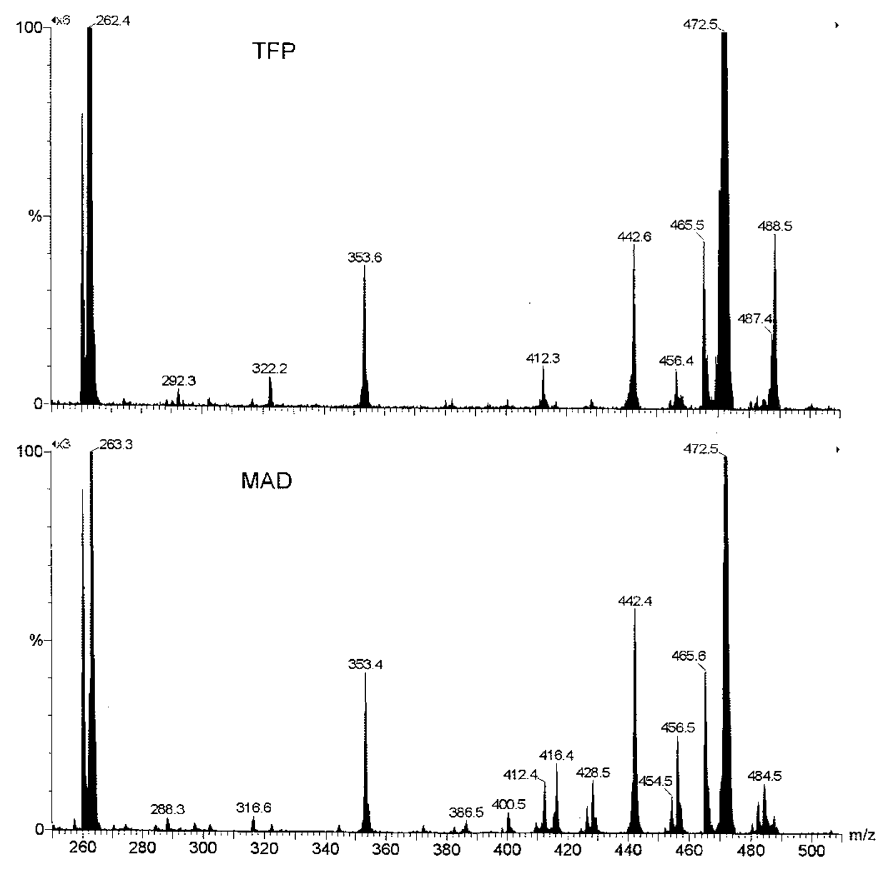

Figure 2. Acylcarnitine intermediate profiles generated by incubation of homogenized fresh muscle with stable isotope labeled palmitate and medium containing L-carnitine. $(A)$ Control; $(B)$ TFP deficiency; $(C)$ MAD deficiency. Ions are designated in the legend to Figure 1. For incubation and analysis conditions see the text.

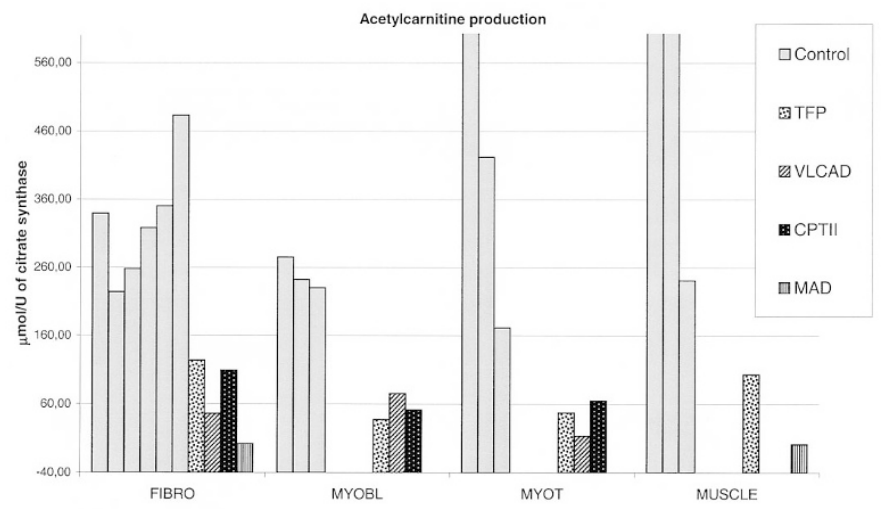

Figure 3. Acetylcarnitine production in control and patient fibroblasts $(F I-$ $B R O)$, myoblasts $(M Y O B L)$, myotubes (MYOT), and skeletal muscle (MUS$C L E$ ) expressed as $\mu \mathrm{mol} / \mathrm{U}$ of citrate synthase.

of harvested cells has been criticized, but clear differences in acylcarnitine profiles in our patient and control cell lines shows that the method is reliable (5).

Although fresh muscle samples are not usually as easily accessible as blood and skin samples, in patients with a neu- 
romuscular presentation, muscle biopsy is often the first tissue sample obtained to screen for potential disorders other than $\beta$-oxidation defects. Analysis of the mitochondrial fatty acid oxidation intermediates from fresh muscle after incubation with palmitate could be the method of choice to diagnose $\beta$-oxidation defects in these patients. The small sample required can easily be taken by needle biopsy. The method may also allow diagnosis of a defect that cannot be detected by analyzing cultured cells namely riboflavin responsive MAD deficiency. The tissue culture media used in culture of fibroblasts contains high concentrations of riboflavin.

Fatty acid oxidation intermediate analysis of cultured and homogenized myoblasts and myotubes produced the similar profile of intermediates as fibroblast or fresh muscle incubations. The higher levels of acetylcarnitine in control muscle compared with control fibroblasts must reflect the differences in either the flux through $\beta$-oxidation or the citric acid cycle. Myoblast culture provides muscle-derived material for further studies and fusion of myoblasts to myotubes allows analysis of muscle cells that resemble more closely the muscle tissue than myoblasts. Although fibroblasts and fresh muscle incubations are more suitable for diagnostic fatty acid oxidation studies, myoblasts, and myotubes could be optimal cell lines for research purposes in patients with neuromuscular manifestations of $\beta$-oxidation disorders.

It seems that most defects of mitochondrial fatty acid oxidation can be reliably detected by incubating cultured cells with labeled or nonlabeled palmitate and carnitine and analyzing produced acylcarnitines by HPLC or MS/MS. Analysis of acylcarnitine intermediates from incubation of fresh skeletal muscle allows rapid diagnosis of a $\beta$-oxidation defect. These analyses of fatty acid oxidation are a welcome addition to diagnosis of $\beta$-oxidation defects. Used together with serum carnitine, blood acylcarnitine, and molecular genetic analyses, these analyses definitively reduce the need for enzyme measurements.

Acknowledgments. The authors thank Rajinder Singh Kler, Ph.D., and Mrs. Christine Hayes for technical assistance.

\section{REFERENCES}

1. Wanders R, Vreken P, den Boer M, Wijburg F, van Gennip A, IJlst L 1999 Disorders of mitochondrial fatty acyl-CoA $\beta$-oxidation. J Inherit Metab Dis 22:442-487

2. Kler R, Jackson S, Bartlett K, Bindhoff L, Eaton S, Pourfarzam M, Frerman F, Goodman S, Watmough N, Turnbull D 1991 Quantitation of acyl-CoA and acylcarnitine esters accumulated during abnormal mitochondrial fatty acid oxidation. J Biol Chem 266:22932-22938

3. Pourfarzam M, Schaefer J, Turnbull DM, Bartlett K 1994 Analysis of fatty acid oxidation intermediates in cultured fibroblasts to detect mitochondrial oxidation disorders. Clin Chem 40:2267-2275

4. Nada MA, Vianeysaban C, Roe CR, Ding JH, Mathieu M, Wappner RS, Bialer MG, Mcglynn JA, Mandon G 1996 Prenatal diagnosis of mitochondrial fatty acid oxidation defects. Prenat Diagn 16:117-124

5. Roe C, Roe D 1999 Recent developments in the investigation of inherited metabolic disorders using cultured human cells. Mol Genet Metab 68:243-257

6. Shen J, Matern D, Millington D, Hillman S, Feezor M, Bennett M, Qumsiyeh M, Kahler S, Chen Y-T, Van Hove J 2000 Acylcarnitines in fibroblasts of patients with long-chain 3-hydroxyacyl-CoA dehydrogenase deficiency and other fatty acid oxidation disorders. J Inherit Metab Dis 23:27-44

7. Ventura F, Costa C, Sruys E, Ruiter J, Allers P, Ijlst L, Tavares de Almeida I, Duran M, Jakobs C, Wanders R 1999 Quantitative acylcarnitine profiling in fibroblasts using [U-13C] palmitic acid: an improved tool for the diagnosis of fatty acid oxidation diagnosis. Clin Chim Acta 281:1-17

8. Schaefer J, Jackson S, Taroni F, Swift P, Turnbull D 1997 Characterisation of carnitine palmitoyltransferases in patients with a carnitine palmitoyltransferase deficiency: implications for diagnosis and therapy. J Neurol Neurosurg Psychiatry 62:169-176

9. Blau H, Webster C 1981 Isolation and characterization of human muscle cells. Proc Natl Acad Sci USA 78:5623-5627

10. Jackson S, Bagstaff S, Lynn S, Yeaman S, Turnbull D, Walker M 2000 Decreased insulin responsiveness of glucose uptake in cultured human skeletal muscle cells from insulin-resistant nondiabetic relatives of type 2 diabetic families. Diabetes 49:11691177

11. Sheperd D, Garland P 1969 Citrate synthase from rat liver. Methods Enzymol 13:11-16 